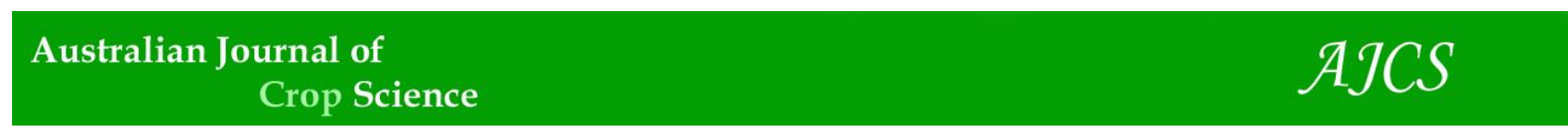

AJCS 11(10):1244-1253 (2017)

ISSN:1835-2707

doi: 10.21475/ajcs.17.11.10.pne473

\title{
Heterosis and path analysis for grain yield and chocolate spot disease resistance in faba bean (Vicia faba L.)
}

\author{
Asnakech Tekalign ${ }^{1,2 *}$, Julia Sibiya ${ }^{1}$, John Derera ${ }^{1}$ \\ ${ }^{1}$ African Centre for Crop Improvement, School of Agricultural, Earth and Environmental Sciences, University \\ of KwaZulu-Natal, P. Bag X01, Scottsville, Pietermaritzburg, South Africa \\ ${ }^{2}$ Ethiopian Institute of Agricultural Research (EIAR), Holetta Agricultural Research Centre (HARC), P.O. Box \\ P.O. Box 2003 Addis Ababa, Ethiopia
}

\section{*Corresponding author: asnakechtek@yahoo.com}

\begin{abstract}
This study was conducted to estimate better-parent and mid-parent heterosis for grain yield and chocolate spot resistance and to determine the direct and indirect effects of yield components on yield of faba bean (Vicia faba L.) in Ethiopia. Ten genetically diverse inbred lines were crossed in a full diallel to produce $90 \mathrm{~F}_{1}$ progenies. The parents and their $90 \mathrm{~F}_{1}$ progenies were evaluated in a $10 \times 10$ alpha lattice design with two replications at three locations. Data were analyzed using the Gardner and Eberhart's analysis II and PATHSAS using SAS program. The maximum heterosis for grain yield $\left(\mathrm{t} \mathrm{ha}^{-1}\right)$ was $162.3 \%$ for mid-parent and $133.9 \%$ over the better parent. Six crosses; NC58 $\times$ ILB-4726, ILB-4726 × Kasa, NC58 × BPL-710, ILB-938 × CS-20-DK, ILB-938 × CS-20-DK and CS-20-DK $\times$ BPL-710 are recommended for grain yield breeding. Similarly, crosses ILB-4726 $\times$ Kasa, ILB-4726 $\times$ Bulga-70, CS-20-DK $\times$ Gebelcho, NC58 $\times$ ILB-4726, Kasa $\times$ BPL-710 and ILB-938 $\times$ Kasa are recommended for chocolate spot resistance breeding. Three crosses ILB-4726 $\times$ Kasa, ILB-4726 $\times$ Bulga-070, NC58 $\times$ ILB-4726 are recommended for both grain yield and chocolate spot disease resistance breeding in faba bean. Path coefficient analysis showed a significant direct effect of the number of nodes that had pods, plant height and total biomass on grain yield. However, general chocolate spot disease score (GDS) and relative area under disease progress curve (rAUDPC) had negative direct effect and significant negative correlation with grain yield. These results are useful to faba bean breeders for indirect selection of grain yield during the early segregating generation when yield tests cannot be conducted.
\end{abstract}

Keywords: Chocolate spot; diallel crosses; faba bean; heterosis; path analysis; yield.

Abbreviations: AUDPC_area under disease progress curve; GDS_general disease score of chocolate spot; rAUDPC_relative area under disease progress curve.

\section{Introduction}

Faba bean (Vicia faba L.) is a good source of protein particularly for the low-income groups in the Middle East and most parts of the Mediterranean, China and Ethiopia (Bond et al., 1985; Haciseferogullari et al., 2003; Ferris and Kaganzi, 2008). It is also a good break crop in cereal production systems for atmospheric nitrogen fixation through symbiosis thus reducing the need for farmers to use artificial fertilizers (Lindemann and Glover, 2003; Farag and Afia, 2012). In Ethiopia, the area for faba bean production increased from 463,174 ha in 2005 to 538,458 ha in 2014 , and the corresponding annual national production increased from 551,984 to 991,700 ton with an average productivity of 1.9 t/ha (FAOSTAT, 2016). Despite its importance, the overall productivity is far behind the potential and the levels needed to offset the increased food demands. Low productivity of faba bean is due to several constraints including lack of high yielding and chocolate spot disease resistant varieties (Gnanasambandam et al., 2012). Efforts have been directed towards improving yield and quality in faba bean in Ethiopia. However, to increase production of faba bean, research should also include identification of adapted high-yielding and chocolate leaf spot resistant genotypes.
Heterosis (hybrid vigour), which is the superiority in performance of a hybrid compared to its parents (Fehr, 1987; Fu et al., 2014) has been reported and widely used in a range of crop species for both self and cross pollinated crops. Expression of heterosis has been investigated for various traits and in identification of genetically divergent populations as base for development of inbred lines to develop crosses with improved characters of interest in faba bean (El-Hady et al., 2006; Alghamdi, 2009), common bean (Tiruneh et al., 2013), wheat (Jatoi et al., 2014), maize (Munaro et al., 2011) and other field crops. Heterosis is described by the trait-specific performance of hybrids relative to the average of its two parents, termed mid-parent heterosis (MPH) or relative to the parent having the best value for the trait, termed best-parent heterosis (BPH) (Duvick, 1999). In faba bean, heterosis for yield has been demonstrated and hybrid varieties have been proposed (Link et al., 1996). Heterosis over mid and better parents were reported in faba bean crosses (Alghamdi, 2009). Zeid et al. (2004) also reported a stable superiority of the hybrids in faba bean over their inbred parents with marked and varying amounts of heterosis. 
Faba bean is a self - pollinating crop though significant outcrossing has been reported ranging from 10 to $80 \%$ with an average of $35 \%$ depending on the environment and genotypes (Bond, 1987; Suso and Moreno, 1999). Thus, populations are highly heterogeneous and considerable potential exists of selecting within populations for specific traits as practiced for disease resistance (Hanounik and Robertson, 1989). Since faba bean is a predominantly self-pollinated crop, commercial products of $F_{1}$ seed are not currently feasible. However, heterosis provides the basis of genetic diversity, guide for choice of desirable parents for developing superior $F_{1}$ progenies to exploit hybrid vigor and building gene pools to be employed in breeding programmes. Moreover, the study of the magnitude of heterosis has a direct effect on the breeding methodology to be followed in varietal improvement.

Component characters may influence yield either jointly or singly and either directly or indirectly through other related characters (Sincik and Goksoy, 2014). Thus, selection based on per se performance without considering the yield component characters may not be effective. Subsequently, understanding of the association of yield component characters with grain yield and among themselves is essential for formulating selection indices. This can be determined through path analysis, which is an efficient statistical technique specially designed to quantify the interrelationships of different components and their direct and indirect effects on yield (Dewey and Lu, 1959).

Path co-efficient values measure the magnitude of direct and indirect effects of characters on complex dependent characters such as yield, and thus enable the breeder to judge the important components during selection. In a study of path analysis between traits and yield, selections based on 1000seed weights and crude protein ratios were recommended due to their significant direct effects on yield in a sunflower breeding programme (Sincik and Goksoy, 2014). Path analysis of yield and its components were examined by different researchers on different crops such as Eruca sativa L. (Jat and Jakhar, 2014), soybean (Garud et al., 2014), mung bean (Dhuppe et al., 2005) and maize (Amini et al., 2013). A significant and direct correlation of plant height with yield was reported in the study of path analysis of faba bean yield and its components (Azarpour et al., 2012).

Grain yield is a complex quantitative character influenced by environmental conditions. Therefore, it is necessary to understand the relationship existing between grain yield and other metric traits of the crop. Such information is limited for faba bean breeding in Ethiopia. Consequently, the objectives of this study were; i) to quantify the magnitude and effect of heterosis for yield and chocolate spot resistance of 10 faba bean genotypes including $90 \mathrm{~F}_{1}$ hybrids from a diallel cross, ii) to investigate the relationships among various yield components, and iii) to determine the direct and indirect effects of other yield component characters on faba bean grain yield.

\section{Results}

\section{Heterosis effects}

Analysis of variance data of the combined diallel cross over the environments for grain yield and chocolate spot disease is presented in Table 1 . The mean squares for variety were highly significant $(\mathrm{P} \leq 0.001)$ for chocolate spot resistance and significant $(\mathrm{P} \leq 0.05)$ for grain yield. The heterosis mean squares were highly significant $(\mathrm{P} \leq 0.001)$ for chocolate spot disease resistance and grain yield. Highly significant $(\mathrm{P} \leq$ 0.001 ) variety heterosis was also observed for chocolate spot resistance and grain yield. Specific heterosis was also significant $(\mathrm{P} \leq 0.001)$ for grain yield.

The estimates for heterosis of parents and $F_{1}$ progenies for grain yield $\left(\mathrm{t} \mathrm{ha}^{-1}\right)$ are presented in Table 2 . A highly significant $(\mathrm{P} \leq 0.001)$ and positive heterosis estimate was observed for parent ILB-4726. On the other hand, Gebecho and Dosha had significant $(\mathrm{P} \leq 0.05)$, negative estimates of heterotic effects for grain yield. Significant $(\mathrm{P} \leq 0.001, \mathrm{P} \leq$ 0.01 and $\mathrm{P} \leq 0.05$ ), positive estimates of specific heterosis effects were recorded in 12 crosses for grain yield (Table 2). Highly significant $(\mathrm{P} \leq 0.001)$ and positive heterotic estimate was recorded for the crosses ILB-4726 $\times$ Bulga-70, NC58 $\times$ BPL-710, ILB-4726 $\times$ Kasa, ILB-938 $\times$ CS-20-DK and BPL$710 \times$ Bulga-70 for grain yield.

\section{Mid-parent and better-parent heterosis for grain yield}

The mid-parent and better-parent heterosis for grain yield are presented in Table 3. The heterosis exhibited by crosses over the respective mid-parent ranged from $-48.5 \%$ (CS-20-DK $\times$ Bulga-70) to $162.3 \%$ (NC58 $\times$ ILB-4726) for grain yield tha ${ }^{1}$. Similarly, heterobeltiosis for grain yield $\mathrm{t} \mathrm{ha}^{-1}$ ranged from $54.1 \%(C S-20-D K \times$ Bulga-70) to $133.9 \%($ NC58 $\times$ ILB4726) over the better parent. Positive heterotic effects are desirable for grain yield. Twenty-two crosses exhibited positive mid-parent heterosis and 15 crosses exhibited positive better-parent heterosis for grain yield $\mathrm{t} \mathrm{ha}^{-1}$. Crosses NC58 $\times$ ILB-4726 (162.3\%) followed by ILB-4726 $\times$ Kasa (148.6\%), ILB-938 $\times$ CS-20-DK (143.6\%), ILB-938 $\times$ CS20-DK (134.6\%), ILB-4726 $\times$ Bulga-70 (129.7\%), and CS20-DK $\times$ BPL-710 (114.2\%) had highest mid-parent heterosis. Similarly, NC58 $\times$ ILB-4726 (133.9\%) had the highest betterparent heterosis, followed by NC58 $\times$ BPL-710 (122.3\%), ILB-4726 $\times$ Kasa (119.3\%), ILB-938 $\times$ CS-20-DK (117.4\%), ILB-938 $\times$ CS-20-DK $(114.1 \%)$ and CS-20-DK $\times$ BPL-710 $(101 \%)$. On the contrary, 23 and 30 crosses had negative mid-parent and better-parent heterosis for grain yield, respectively. The crosses involved; CS-20-DK $\times$ Bulga-70, ILB-938 $\times$ BPL-710 and NC58 $\times$ Bulga-70 had $-48.5 \%$, $46.8 \%,-46.9 \%$ for mid-parent heterosis, respectively and CS20-DK $\times$ Bulga-70, NC58 $\times$ Bulga-70 and BPL-710 $\times$ Gebelcho with $-54.1,-53.3$, and -50.7 better-parent heterosis, respectively.

\section{Mid-parent and better-parent heterosis for chocolate spot disease resistance}

The heterosis over the mid-parent and better-parent for chocolate spot disease resistance are presented in Table 4. The magnitude of heterosis over the respective mid-parent ranged from $-29.39 \%$ (ILB-4726 $\times$ Kasa) to $116.35 \%$ (ILB$938 \times$ ILB-4726) for chocolate spot disease resistance based on the general disease score (GDS) and from $-43.2 \%$ (ILB$4726 \times \mathrm{CS}-20-\mathrm{DK})$ to $176.3 \%$ (ILB-938 $\times$ ILB-4726) for relative area under disease progress curve (rAUDPC). Heterobeltiosis for chocolate spot disease resistance ranged from $-60.5 \%$ (ILB-4726 $\times$ Kasa) to $111.1 \%$ (ILB-938 $\times$ ILB4726) over better-parent. Negative heterotic effects are desirable for chocolate spot disease resistance. Twenty-nine crosses exhibited negative heterosis over mid-parent ranging from -29.4 to -4.56 and forty-one crosses exhibited negative heterobeltiosis over better-parent ranging from -60.5 to -4.5 based on the GDS. Similarly, 29 crosses exhibited negative heterosis over mid-parent ranging from -21.4 to -1.2 and 42 crosses exhibited negative heterosis over better-parent ranging from -43.2 to -1.8 based on the rAUDPC. 
Table 1. Combined analysis of variance of heterosis for grain yield and chocolate spot disease in the diallel over three locations in the diallel cross

\begin{tabular}{|c|c|c|c|c|}
\hline Source of variation & df & GDS & rAUDPC & GY \\
\hline Env & 2 & $1487.2 * * *$ & $1045481.8^{* * *}$ & $124.02 * * *$ \\
\hline REP(Env) & 3 & $130.0^{* * *}$ & $217250.6^{* * *}$ & $6.03 * *$ \\
\hline Entry & 54 & $160.9^{* * *}$ & 107161.1 *** & $12.89 * * *$ \\
\hline Env*Entry & 108 & $15.2^{*}$ & $17899.6^{* *}$ & $1.90^{* * *}$ \\
\hline Variety SS & 9 & $851.93 * * *$ & $389309.84 * * *$ & $3.13^{*}$ \\
\hline Heterosis SS & 45 & $22.80 * * *$ & $50731.34 * * *$ & $14.84 * * *$ \\
\hline Average HET SS & 1 & 2.63 & 48.9 & 10.56 \\
\hline Variety HET SS & 9 & $45.89 * * *$ & $173197.52^{* * * *}$ & $7.87 * * *$ \\
\hline Specific HETEROSIS & 35 & 17.44 & 20688.11 & $16.757 * * *$ \\
\hline Variety x Env & 18 & $30.09 * * *$ & $33923.01 * * *$ & 1.36 \\
\hline Heterosis $\mathrm{x}$ Env & 90 & 12.27 & 14694.88 & $2.01 * * *$ \\
\hline Average HET x Env & 2 & 13.53 & $77617.09 * * *$ & $9.55 * * *$ \\
\hline Variety HET x Env & 18 & 9.89 & 12733.67 & 1.310 \\
\hline Specific HET x Env & 70 & 12.84 & 13401.41 & $1.98 * * *$ \\
\hline Error & & 11.27 & 11352 & 1.0935 \\
\hline $\mathrm{CV}$ & & 21 & 21 & 30 \\
\hline $\mathrm{R}^{2}$ & & 88.2 & 85 & 86.8 \\
\hline Mean & & 15.4 & $0.29(503.6)$ & 3.460 \\
\hline
\end{tabular}

$*, * *, * * *$ Significant at $0.05,0.01$, and 0.001 probability levels, respectively; $\mathrm{R}^{2}$ : coefficient of determination; $\mathrm{CV}$ : coefficient of variation; rAUDPC: relative area under disease progress curve number in parenthesis is mean for AUDPC

Table 2. Estimate of parent heterosis (diagonal) and specific heterosis of the cross for grain yield $\left(\mathrm{t} \mathrm{ha}^{-1}\right)$ across the three locations.

\begin{tabular}{|c|c|c|c|c|c|c|c|c|c|c|}
\hline & Moti & ILB-938 & NC58 & Dosha & ILB-4726 & CS-20-DK & Kasa & BPL-710 & Gebelcho & Bulga-70 \\
\hline Moti & -0.676 & $1.32^{* *}$ & 0.292 & -0.626 & 0.052 & 0.152 & -0.307 & -0.072 & 0.036 & -0.844 \\
\hline ILB-938 & & 0.528 & $1.14 *$ & -0.006 & $-2.6^{* * * *}$ & $1.89 * * *$ & 0.72 & $-2.56 * * *$ & $-1.26^{*}$ & $1.36^{* *}$ \\
\hline NC58 & & & 0.317 & $-1.15^{*}$ & $1.48 * *$ & $-2.11 * * *$ & $-1.74 * *$ & $2.61^{* * *}$ & $1.45^{* * *}$ & $-1.99 * * *$ \\
\hline Dosha & & & & $-0.906 *$ & 0.351 & -0.634 & 0.564 & 0.196 & $1.56 * *$ & -0.26 \\
\hline ILB-4726 & & & & & $1.378 * * *$ & $1.29 *$ & $2.03 * * *$ & $-2.98 * * *$ & $-2.25 * * *$ & $2.63 * * *$ \\
\hline CS-20-DK & & & & & & 0.166 & $-1.67 * *$ & $1.81^{* *}$ & 1.027 & $-1.76^{* *}$ \\
\hline Kasa & & & & & & & -0.086 & 0.787 & 0.787 & $-1.18^{*}$ \\
\hline BPL-710 & & & & & & & & 0.361 & -1.59 ** & $1.81^{* * *}$ \\
\hline Gebelcho & & & & & & & & & $-0.785^{*}$ & 0.232 \\
\hline Bulga-70 & & & & & & & & & & -0.297 \\
\hline
\end{tabular}

Bulga-70

Table 3. Percent mid-parent heterosis (below diagonal) and better-parent heterosis (above diagonal) of the $90 \mathrm{~F}_{1}$ progenies for grain yield $\left(\mathrm{t}\right.$ ha ${ }^{-1}$ ) across the three locations in the diallel cross.

\begin{tabular}{|c|c|c|c|c|c|c|c|c|c|c|}
\hline & Moti & ILB-938 & NC58 & Dosha & ILB-4726 & CS-20-DK & Kasa & BPL-710 & Gebelcho & Bulga-70 \\
\hline Moti & & 10.84 & -21.44 & -38.73 & 11.91 & -34.76 & -37.85 & -24.43 & -39.47 & -42.61 \\
\hline ILB-938 & 49.01 & & 87.99 & -18.16 & 11.91 & 117.38 & 59.00 & -47.70 & -35.68 & 42.29 \\
\hline NC58 & 1.24 & 99.63 & & -47.62 & 133.96 & -44.32 & -37.83 & 122.29 & -2.04 & -53.33 \\
\hline Dosha & -34.60 & 5.05 & -35.80 & & -2.16 & -21.90 & -27.68 & -17.20 & -2.02 & -37.16 \\
\hline ILB-4726 & 56.22 & -17.96 & 162.29 & 30.86 & & 119.32 & -42.18 & -45.57 & 82.88 & 114.06 \\
\hline CS-20-DK & -16.97 & 134.58 & -43.36 & -5.58 & 143.63 & & -27.29 & 101.04 & -3.15 & -54.09 \\
\hline Kasa & -20.74 & 71.13 & -36.94 & -12.36 & 148.98 & -27.08 & & -9.89 & -48.02 & 69.01 \\
\hline BPL-710 & 0.68 & -46.99 & 133.04 & 5.23 & -37.95 & 114.23 & 79.61 & & -50.73 & 49.81 \\
\hline Gebelcho & -34.62 & -18.20 & 18.87 & -0.77 & -27.83 & 15.92 & 8.11 & -37.97 & & -23.45 \\
\hline Bulga-70 & -33.68 & 70.66 & -46.84 & -31.55 & 129.69 & -48.48 & -41.52 & 77.72 & -17.59 & \\
\hline
\end{tabular}




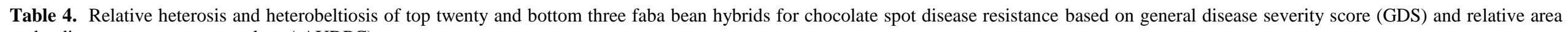
under disease progress curve values (rAUDPC).

\begin{tabular}{|c|c|c|c|c|c|}
\hline \multirow[b]{2}{*}{$\mathrm{F}_{1}$ progenies } & \multicolumn{5}{|c|}{ Chocolate spot disease (\%) } \\
\hline & MP-GDS & MP-rAUDPC & $F_{1}$ progenies & BP-GDS & BP-rAUDPC \\
\hline ILB-4726 x Kasa & -29 & -4.46 & ILB-4726 x Kasa & -60.52 & -38.60 \\
\hline CS-20-DK x Gebelcho & -28 & -18.86 & ILB-47 26 x Bulga-70 & -55.28 & -37.54 \\
\hline Kasa x Bulga-70 & -26 & -16.51 & ILB-938 x Kasa & -55.07 & -27.30 \\
\hline Dosha x Gebelcho & -23 & -20.25 & NC58 x ILB- 4726 & -49.28 & -39.45 \\
\hline Dosha x Kasa & -23 & -14.71 & ILB-4726 x CS-20-DK & -49.11 & -43.20 \\
\hline ILB-47 26 x Bulga-70 & -23 & -5.08 & Kasa x BPL-710 & -47.31 & -11.94 \\
\hline ILB-938 x Kasa & -20 & -20.84 & ILB-938 x NC58 & -45.05 & -27.92 \\
\hline CS-20-DK x Bulga-70 & -19 & -15.98 & BPL-710 $\times$ Bulga-70 & -44.96 & -24.56 \\
\hline Kasa x Gebelcho & -19 & -8.62 & CS-20-DK x BPL-710 & -44.50 & -27.97 \\
\hline NC58 x Bulga-70 & -19 & -9.76 & ILB-938 x CS-20-DK & -44.34 & -26.52 \\
\hline Moti x Kasa & -17 & -10.55 & NC58 x BPL-710 & -43.46 & -29.68 \\
\hline NC58 x CS-20-DK & -15 & -17.15 & CS-20-DK x Gebelcho & -41.14 & -29.44 \\
\hline CS-20-DK x Kasa & -14 & -1.95 & Kasa x Gebelcho & -40.64 & -20.53 \\
\hline NC58 x Dosha & -14 & -13.86 & Dosha x Kasa & -40.20 & -21.70 \\
\hline Dosha x CS-20-DK & -13 & -15.59 & Moti x Kasa & -37.13 & -14.99 \\
\hline ILB-4726 x CS-20-DK & -12 & -3.36 & Dosha x ILB-4726 & -37.02 & -24.90 \\
\hline NC58 x Kasa & -12 & 1.04 & Kasa x Bulga-70 & -34.53 & -19.45 \\
\hline NC58 x Gebelcho & -12 & -4.25 & Dosha x BPL-710 & -32.20 & -18.09 \\
\hline NC58 x ILB-4726 & -12 & 2.18 & ILB-938 x Gebelcho & -31.85 & -22.32 \\
\hline Moti x CS-20-DK & -12 & -17.77 & ILB-938 x Bulga-70 & -31.67 & -7.72 \\
\hline ILB-4726 x BPL-710 & 79 & 120.89 & ILB-4726 x BPL-710 & 52.23 & 74.64 \\
\hline ILB-938 x BPL-710 & 106 & 164.67 & ILB-938 x BPL-710 & 79.49 & 121.41 \\
\hline ILB-938 xILB-4726 & 116 & 196.53 & ILB-938 xILB-4726 & 111.08 & 176.28 \\
\hline
\end{tabular}

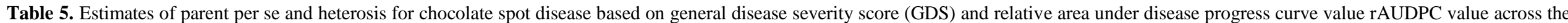
three locations in the diallel cross.

\begin{tabular}{|c|c|c|c|c|}
\hline & \multicolumn{2}{|c|}{ Estimate of GDS } & \multicolumn{2}{|c|}{ Estimate of rAUDPC } \\
\hline Parameter & Per se & Heterosis GDS & Per se & Heterosis rAUDPC \\
\hline Moti & 2.87 & 0.74 & 79.96 & $76.29 *$ \\
\hline ILB-938 & $-11.79 * * *$ & $2.51^{*}$ & $-362.32 * * *$ & $152.70^{* * * *}$ \\
\hline NC58 & $7.94 * * *$ & -1.76 & $346.5 * * *$ & $-166.35^{* * * *}$ \\
\hline Dosha & 2.09 & -1.07 & 79.92 & -31.04 \\
\hline ILB-4726 & $-11.60 * * *$ & 0.43 & $-341.72 * * *$ & 47.02 \\
\hline CS-20-DK & $7.85^{* * *}$ & $2.33^{*}$ & $176.50^{* *}$ & -63.17 \\
\hline Kasa & $6.64 * * *$ & 0.88 & $326.77 * * *$ & $98.24 * *$ \\
\hline BPL-710 & $-10.19 * * *$ & $2.39 *$ & $-293.32 * * *$ & $145.25^{* * *}$ \\
\hline Gebelcho & -0.77 & -1.61 & 12.24 & -50.56 \\
\hline Bulga-70 & $6.94 * * *$ & -0.17 & $135.37 *$ & -11.90 \\
\hline
\end{tabular}




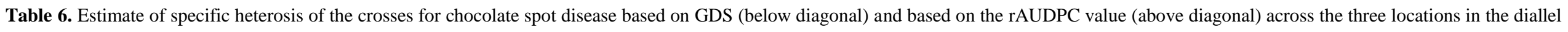
cross.

\begin{tabular}{|c|c|c|c|c|c|c|c|c|c|c|}
\hline & Moti & ILB-938 & NC58 & Dosha & ILB-4726 & CS-20-DK & Kasa & BPL-710 & Gebelcho & Bulga-70 \\
\hline Moti & & 8.89 & $-107.24 *$ & 61.58 & 45.76 & -42.99 & -21.86 & 48.35 & -24.73 & 32.23 \\
\hline ILB-938 & 0.42 & & -42.43 & 36.34 & 12.5 & -1.7 & -61.57 & 18.15 & -44.7 & 74.52 \\
\hline NC58 & -2.46 & -0.4 & & 68.78 & 48.37 & -14.07 & 47.75 & $-106.99^{*}$ & 95.95 & 9.87 \\
\hline Dosha & 1.29 & -1.1 & 1.34 & & 18.95 & -3.32 & -104.56 & -29.19 & -60.45 & 11.86 \\
\hline ILB-4726 & 0.02 & 1.39 & 0.06 & 0.91 & & -66.3 & -42.65 & 12.74 & 54.17 & -83.54 \\
\hline CS-20-DK & -0.95 & 0.24 & 0.48 & 1.77 & -1.41 & & 96.05 & 62.57 & -35.32 & 5.08 \\
\hline Kasa & -0.59 & -1.87 & 2.33 & -2.42 & -1.54 & 1.99 & & 35.81 & 42.38 & 8.66 \\
\hline BPL-710 & 0.65 & 0.85 & -2.91 & -1.18 & 2.15 & 0.69 & 0.83 & & -5.03 & -36.41 \\
\hline Gebelcho & -0.26 & -1.28 & 2.55 & -1.61 & 1.42 & -3 & 1.31 & 0.32 & & -22.28 \\
\hline Bulga-70 & 1.88 & 1.75 & -0.99 & 1.02 & -2.97 & 0.19 & -0.03 & -1.4 & 0.55 & \\
\hline
\end{tabular}

Table 7. Direct effect (along diagonal in bold), indirect effect (off diagonals) and total correlation (at the end in bold) of yield component characters and chocolate spot disease on grain yield ( $t$ ha' ${ }^{1}$ ) in the $90 \mathrm{~F}_{1}$ progenis in the diallel crosses.

\begin{tabular}{|c|c|c|c|c|c|c|c|c|c|c|c|c|c|c|c|c|}
\hline & DTF & DTM & $\mathrm{BB}$ & HFP & NPPN & NPPP & NSPP & NNP & NWP & $\mathrm{PH}$ & HSW & BM & rAUDPC & GDS & $\mathrm{HI}$ & GY \\
\hline DTF & 0.00 & 0.05 & -0.03 & 0.03 & 0.00 & 0.00 & 0.00 & 0.00 & -0.02 & $\begin{array}{l}-0.01 \\
\end{array}$ & 0.00 & 0.17 & -0.03 & 0.11 & -0.31 & -0.04 \\
\hline DTM & 0.00 & 0.06 & -0.11 & 0.03 & 0.01 & -0.01 & 0.00 & 0.01 & 0.14 & 0.00 & 0.01 & 0.37 & -0.02 & 0.12 & -0.37 & $0.24 *$ \\
\hline BB & 0.00 & 0.02 & $-0.36 * * *$ & 0.01 & 0.02 & -0.03 & 0.03 & 0.04 & 0.46 & 0.01 & 0.01 & 0.67 & -0.01 & 0.04 & -0.20 & $0.72 * * *$ \\
\hline HFP & 0.00 & 0.05 & -0.09 & 0.04 & 0.00 & 0.00 & 0.00 & 0.01 & 0.08 & 0.00 & 0.01 & 0.28 & -0.02 & 0.11 & -0.33 & 0.13 \\
\hline NPPN & 0.00 & 0.01 & -0.33 & 0.01 & 0.03 & -0.03 & 0.03 & 0.05 & 0.53 & 0.01 & 0.01 & 0.66 & -0.01 & 0.03 & -0.17 & $0.83^{* * *}$ \\
\hline NPPP & 0.00 & 0.02 & -0.29 & 0.00 & 0.02 & -0.04 & 0.03 & 0.05 & 0.53 & 0.01 & 0.01 & 0.62 & 0.00 & 0.03 & -0.12 & $0.88^{* * *}$ \\
\hline NSPP & 0.00 & 0.01 & -0.29 & 0.00 & 0.02 & -0.03 & 0.04 & 0.05 & 0.48 & 0.00 & 0.01 & 0.55 & -0.01 & 0.02 & -0.11 & $0.76^{* * *}$ \\
\hline NNP & 0.00 & 0.01 & -0.27 & 0.01 & 0.02 & -0.03 & 0.04 & 0.06 & 0.48 & 0.00 & 0.01 & 0.55 & 0.00 & 0.03 & -0.14 & $0.75^{* * *}$ \\
\hline NWP & 0.00 & 0.02 & -0.30 & 0.01 & 0.02 & -0.03 & 0.04 & 0.05 & $0.56^{* * * *}$ & 0.01 & 0.01 & 0.63 & -0.01 & 0.03 & -0.13 & $0.90^{* * *}$ \\
\hline PH & 0.00 & 0.00 & -0.04 & 0.00 & 0.00 & -0.01 & 0.00 & 0.00 & 0.09 & $0.07 *$ & 0.00 & 0.14 & 0.00 & 0.01 & 0.01 & $0.28^{* *}$ \\
\hline HSW & 0.00 & 0.03 & -0.33 & 0.02 & 0.02 & -0.03 & 0.03 & 0.04 & 0.45 & 0.01 & 0.01 & 0.68 & -0.02 & 0.07 & -0.24 & $0.74 * * *$ \\
\hline BM & 0.00 & 0.03 & -0.32 & 0.01 & 0.02 & -0.03 & 0.03 & 0.04 & 0.47 & 0.01 & 0.01 & $0.75^{* * *}$ & -0.01 & 0.06 & -0.27 & $0.82^{* * *}$ \\
\hline rAUDPC & 0.00 & -0.04 & 0.11 & -0.03 & 0.00 & 0.00 & -0.01 & -0.01 & -0.08 & 0.01 & -0.01 & -0.26 & -0.04 & -0.13 & 0.25 & $-0.15^{*}$ \\
\hline GDS & 0.00 & -0.05 & 0.11 & -0.03 & -0.01 & 0.01 & -0.01 & -0.01 & -0.11 & 0.00 & -0.01 & -0.32 & 0.03 & -0.15 & 0.31 & $-0.23^{*}$ \\
\hline HI & 0.00 & -0.05 & 0.15 & -0.03 & -0.01 & 0.01 & -0.01 & -0.02 & -0.16 & 0.00 & -0.01 & -0.42 & 0.02 & -0.09 & $0.48^{* * *}$ & -0.13 \\
\hline
\end{tabular}

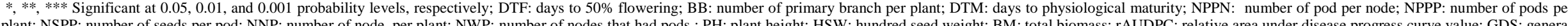
disease severity score; HI: harvest index ; GY: grain yield. 
Table 8. Correlation coefficients for grain yield $\left(\mathrm{t} \mathrm{ha}^{-1}\right)$, yield components and chocolate spot disease for the $90 \mathrm{~F}_{1}$ progenies in the diallel cross.

\begin{tabular}{|c|c|c|c|c|c|c|c|c|c|c|c|c|c|c|c|c|}
\hline & DTF & DTM & BB & HFP & NPPN & NPPP & NSPP & NNP & NWP & $\mathrm{PH}$ & HSW & $\mathrm{BM}$ & rAUDPC & GDS & GY & $\mathrm{HI}$ \\
\hline DTF & 1 & $0.78 * * *$ & 0.07 & $0.71 * * *$ & -0.02 & -0.02 & -0.09 & 0.01 & -0.03 & -0.17 & $0.21 *$ & $0.23^{*}$ & $-0.66 * * *$ & $-0.75 * * *$ & -0.04 & $-0.65 * * *$ \\
\hline DTM & & 1 & $0.30^{* * *}$ & $0.76 * * *$ & $0.24 *$ & $0.25^{*}$ & 0.12 & $0.22 *$ & $0.25^{*}$ & 0.001 & $0.43^{* * * *}$ & $0.50^{* * * *}$ & $-0.63 * * *$ & $-0.81 * * *$ & $0.24 *$ & $-0.78 * * *$ \\
\hline BB & & & 1 & $0.24 *$ & $0.91 * * *$ & $0.80 * * *$ & $0.82 * * *$ & $0.76 * * *$ & $0.82 * * *$ & 0.11 & $0.92^{* * * *}$ & $0.89^{* * *}$ & $-0.30 * *$ & $-0.32 * *$ & $0.72^{* * * *}$ & $-0.43 * * *$ \\
\hline HFP & & & & 1 & 0.17 & 0.11 & 0.08 & 0.17 & 0.14 & 0.05 & $0.39^{* * *}$ & $0.38^{* * * *}$ & $-0.64 * * *$ & $-0.75^{* * *}$ & 0.13 & $-0.70 * * *$ \\
\hline NPPN & & & & & 1 & $0.91 * * *$ & $0.85 * * *$ & $0.83 * * *$ & $0.94 * * *$ & 0.16 & $0.85^{* * * *}$ & $0.89^{* * * *}$ & -0.15 & $-0.21 *$ & $0.83^{* * * *}$ & $0.36^{* * * *}$ \\
\hline NPPP & & & & & & 1 & $0.85 * * *$ & $0.83 * * *$ & $0.96 * * *$ & 0.18 & $0.77 * * *$ & $0.83^{* * *}$ & -0.11 & $-0.19 *$ & $0.88^{* * * *}$ & $-0.25^{*}$ \\
\hline NSPP & & & & & & & 1 & $0.91 * * *$ & $0.87 * * *$ & -0.02 & $0.78^{* * * *}$ & $0.74 * * * *$ & -0.14 & -0.15 & $0.76^{* * * *}$ & $-0.22 *$ \\
\hline NNP & & & & & & & & 1 & $0.85 * * *$ & 0.01 & $0.73 * * * *$ & $0.74 * * *$ & -0.13 & $-0.21 *$ & $0.75^{* * * *}$ & $-0.31 * *$ \\
\hline NWP & & & & & & & & & 1 & 0.16 & $0.81 * * *$ & $0.85^{* * * *}$ & -0.14 & $-0.20 *$ & $0.90 * * *$ & $-0.28 * *$ \\
\hline PH & & & & & & & & & & 1 & 0.13 & $0.19^{*}$ & 0.11 & -0.04 & $0.28^{* *}$ & 0.019 \\
\hline HSW & & & & & & & & & & & 1 & $0.91 * * *$ & $-0.46 * * *$ & $-0.47 * * *$ & $0.74 * * *$ & $-0.51 * * *$ \\
\hline $\mathrm{BM}$ & & & & & & & & & & & & 1 & $-0.35 * * *$ & $-0.43 * * *$ & $0.82 * * *$ & $-0.56 * * *$ \\
\hline rAUDPC & & & & & & & & & & & & & 1 & $0.87 * * *$ & $-0.18 *$ & $0.52 * * *$ \\
\hline GDS & & & & & & & & & & & & & & 1 & $-0.23 *$ & $0.65 * * *$ \\
\hline GY & & & & & & & & & & & & & & & 1 & -0.13 \\
\hline $\mathrm{HI}$ & & & & & & & & & & & & & & & & 1 \\
\hline
\end{tabular}
*, ***** Significant at $0.05,0.01$, and 0.001 probability levels, respectively; DTF: days to $50 \%$ flowering; DTM: days to physiological maturity; NPPN: number of pod per node; NPPP: number of pods per plant; NSPP: number of seeds per pod;
NNP: number of node per plant; NWP: number of nodes that had pods ; PH: plant height; HSW: hundred seed weight; BM: total biomass; rAUDPC: relative area under disease progress curve value; GDS: general disease severity score; GY: grain
yield ; HI: harvest index.

Table 9. Description of the parental inbred lines used for $10 \mathrm{x} 10$ diallel crosses of faba bean.

\begin{tabular}{|c|c|c|c|c|c|c|}
\hline \multirow[b]{2}{*}{ Parent lines } & \multirow[b]{2}{*}{ Pedigree } & \multirow[b]{2}{*}{${ }^{{ }^{a} \text { Genotypic cluster group }}$} & \multicolumn{3}{|c|}{ Estimate of GCA effect for } & \multirow[b]{2}{*}{ Source } \\
\hline & & & Resistant level to $\mathrm{CH}$ & $\mathrm{CH}$ resistance & Grain yield & \\
\hline ILB-4726 & ICARDA & I & Resistant & Negative & Positive & ICARDA \\
\hline ILB-938 & ICARDA & I & Resistant & Negative & Positive & ICARDA \\
\hline BPL-710 & ICARDA & I & Resistant & Negative & Negative & ICARDA \\
\hline Moti & ILB-4432 x kuse-2-27-33 & II & Moderately resistant & Positive & Positive & HARC \\
\hline Dosha & Landrace collection & II & Moderately resistant & Positive & Negative & HARC \\
\hline Gebelcho & Tesfa x ILB-4726 & II & Moderately resistant & Negative & Negative & HARC \\
\hline Kasa & Landrace collection & Il & Susceptible & Positive & Negative & HARC \\
\hline NC58 & National collection & III & Susceptible & Positive & Negative & HARC \\
\hline CS-20-DK & National collection & III & Susceptible & Positive & Positive & HARC \\
\hline Bulga-70 & Landrace collection & III & Susceptible & Positive & Negative & HARC \\
\hline
\end{tabular}

${ }^{a}$ Genotypic cluster group indicated the cluster group of the parent lines based on the result of molecular analysis using SSR markers; ICARD; International Center for Agricultural Research in the Dry Area; HARC; Holetta Agricultural Research Center;

GCA General combining ability; $\mathrm{CH}$ : Chocolate spot disease 
The best hybrids with marked performance in negative direction for chocolate spot resistance were ILB-4726 $\times$ Kasa, CS-20-DK $\times$ Gebelcho, Kasa $\times$ Bulga-70, Dosha $\times$ Gebelcho, Dosha $\times$ Kasa and ILB-4726 $\times$ Bulga-70 with $-29.4 \%$, $27.9 \%,-25.7 \%,-23.3 \%,-22.8 \%$ and $-22.6 \%$ mid-parent heterosis, respectively (Table 4). These crosses also recorded negative direction performance for mid-parent heterosis based on the rAUDPC. Likewise, hybrids ILB-4726 $\times$ Kasa, ILB-4726 $\times$ Bulga-70 had marked performance in negative direction ILB-938 $\times$ Kasa, NC58 $\times$ ILB-4726, and Kasa $\times$ BPL-710 with $-60.5 \%,-55.3 \%,-55.1 \%,-49.3 \%,-49.1 \%$ and $47.3 \%$ better-parent heterosis, respectively. Similar performance in negative direction and magnitude were also recorded for these hybrids based on the rAUDPC disease assessment. On the contrary, the hybrids ILB-4726 $\times$ BPL710, ILB-938 $\times$ BPL-710 and ILB-4726 $\times$ BPL-710 performed in positive direction for chocolate spot resistance with high magnitude of mid-parent and better-parent heterosis based on both GDS and rAUDPC value assessments (Table 4).

The estimate of parent per se and heterosis effect and estimate of specific heterosis effect of the $F_{1}$ progenies for chocolate spot disease resistance are presented in Table 5 and 6. Highly significant $(\mathrm{P} \leq 0.001)$ and negative estimate per se were recorded for the genotypes ILB-938, ILB-4726 and BPL-710 based on both GDS and rAUDPC assessments

(Table 5). Nonetheless, significant $(\mathrm{P} \leq 0.01)$ and positive estimate of heterotic effect was observed for these three parents for chocolate spot resistance based on both GDS and rAUDPC assessments. Negative estimates of specific heterosis effects were observed on 19 and 21 crosses for chocolate spot resistance based on GDS and rAUDPC assessments, respectively (Table 6).

Path coefficient analysis of grain yield $\left(\mathrm{t} \mathrm{ha}^{-1}\right)$, chocolate spot disease and yield components in faba bean progenies from a diallel cross

The direct and indirect effects of yield component characters along with their correlation coefficients with grain yield are presented in Table 7 and 8 . The results of path coefficient analysis based on grain yield as a dependent variable revealed high significant $(\mathrm{P} \leq 0.001)$ and positive direct effects of total biomass (0.75), number of nodes that had pods $(0.56)$ and harvest index (0.48) on grain yield (Table 7). However, there was high significant $(\mathrm{P} \leq 0.001)$ and negative direct effects of number of primary branches on grain yield. Chocolate spot disease had negative direct effects on grain yield. The results of indirect effects indicated that hundred seed weight (0.68), number of primary branch (0.67), number of pods per plant (0.62), number of nodes that had pods $(0.63)$, number of seeds per pod (0.55) and number of nodes per plant (0.55) had positive indirect effects on grain yield, which could be justified through the total biomass. Similarly, number of pods per node (0.53), number of pods per plant $(0.53)$, number of seeds per pod (0.48), number of nodes per plant (0.48), primary branches $(0.46)$, and hundred seed weight $(0.46)$ had positive indirect effects on grain yield which could be justified via the number of nodes that had pods (0.56). Consequently, it can be explained that chocolate spot disease $(-0.32)$ and rAUDPC $(-0.26)$ had indirect and negative effects on grain yield via the total biomass. Similarly, days to flowering, chocolate spot disease (GDS and rAUDPC) and harvest index had indirect and negative effects on grain yield (Table 7).
The path coefficient analysis resulted in high significant $(\mathrm{P} \leq$ 0.001 ), positive correlation of grain yield with number of nodes that had pods $(\mathrm{r}=0.90)$, number of pods per plant $(\mathrm{r}=0.88)$, number of pods per node $(\mathrm{r}=0.83)$, total biomass $(\mathrm{r}=0.82)$, number of seeds per pod $(\mathrm{r}=0.76)$, number of nodes per plant $(\mathrm{r}=0.75)$, hundred seed weight $(\mathrm{r}=0.74)$, and primary branch $(\mathrm{r}=0.72)$. Conversely, there was significant $(\mathrm{P} \leq 0.05)$ and negative correlation between yield and chocolate spot disease assessments (Table 7 and 8 ). There was high significant $(\mathrm{P} \leq 0.001)$ positive correlation among most of the yield component characters (Table 8 ).

\section{Discussion}

The mean squares due to the variety were significant for chocolate spot resistance and grain yield indicating that the parents were diverse and from different genetic backgrounds. Similarly, the significant mean squares due to heterosis, variety heterosis and specific heterosis for chocolate spot disease resistance and grain yield suggested the potential of the parents for heterosis. These results confirm those obtained by Zeid et al. (2004) and Alghamdi (2009).

The heterosis percentage for grain yield relative to the midparent and better-parent were positive for 22 and 15 crosses, respectively. The highest heterosis for grain yield of $162.3 \%$ in this study was higher than reported in other studies, for instance $129.9 \%$ by Alghamdi (2009), $119 \%$ by (Zeid et al., 2004). The high heterosis for grain yield was detected in crosses made between the introduced inbred lines (ILB-4726, ILB-938 and BPL-710) and locally released improved varieties (NC58, Kasa, Bulga-70 and CS-20-DK). This suggested these lines were genetically divergent and these parents were from different clusters. Das et al. (2013) reported a positive correlation between genetic divergence and heterosis. This is also explained by line ILB-4726, ILB938 and BPL-710, which had estimates of heterosis effects in the positive direction for grain yield.

In this study, the crosses NC58 $\times$ ILB-4726, ILB-4726 $\times$ Kasa, NC58 $\times$ BPL-710, ILB-938 $\times$ CS-20-DK, ILB-938 $\times$ CS-20-DK and CS-20-DK $\times$ BPL-710 had the highest midparent and better-parent heterosis for grain yield. In addition, highly significant estimates and positive heterotic effects for grain yield were recorded for the specific heterotic crosses made between the genetically divergent lines, for instance for crosses; ILB-4726 × Bulga-70, NC58 × BPL-710, ILB-4726 $\times$ Kasa, ILB-938 $\times$ CS-20-DK and BPL-710 $\times$ Bulga-70. The negative heterosis was found between crosses of genetically close inbred lines. For instance crosses between; CS-20-DK and Bulga-70, ILB-938 and BPL-710, NC58 and Bulga70 which had a high degree of parental relationship genetically had negative heterosis for grain yield over both the mid and better-parent.

The desirable negative heterosis for chocolate spot disease resistance was obtained in crosses made between the inbred lines: ILB-4726, ILB-938 and BPL-710, Gebelco and NC58, Kasa, Bulga-70 and CS-20-DK. This could be due to lines ILB-4726, ILB-938, BPL-710 and Gebelcho, which had estimates of heterosis effect in the negative direction for chocolate spot disease resistance. In this study, the best hybrids which showed heterosis in the negative direction over the mid and better-parent for chocolate spot resistance were between resistant lines $\mathrm{x}$ susceptible ones; ILB-4726 $\times$ Kasa, ILB-4726 × Bulga-70, CS-20-DK ×x Gebelcho, NC58 $\times$ ILB-4726, Kasa $\times$ BPL-710 and ILB-938 $\times$ Kasa. This is also explained by the genetic divergence of the parental lines. On the contrary, positive heterotic effects were found between resistant lines x resistant lines: ILB-4726 × BPL-710, ILB- 
$938 \times$ BPL-710 and ILB-4726 $\times$ BPL-710 which performed in positive direction for chocolate spot resistance. This could be due to ILB-4726, ILB-938, BPL-710 and Gebelcho (its pedigree comprises ILB-4726) which are genetically close inbred lines. Matiello et al. (2012) and Lim and White (1978) reported similar results of estimates of heterosis for maize anthracnose disease and Colletotrichum graminicola resistance in maize, respectively. Path analysis helps in measuring the direct effect of each trait as well as its indirect effect through other characters contributing to yield. In the present study, the total biomass followed by number of nodes that had pods, had the highest positive direct effect on grain yield $t \mathrm{ha}^{-1}$.

This implied that high grain yielding varieties had high total biomass, high number of nodes that had pods and high harvest index. This finding substantiates the result in the study of path analysis on some faba bean landraces from Ethiopia by Tadesse et al. (2011) where the number of seeds per pods and thousand seed weight had direct effect on yield. Similarly, Farshadfar and Farshadfar (2008) also reported that pod number had the highest direct effect on yield of chickpea. The results of indirect effects indicated that most of the yield component characters except chocolate spot disease and days to flowering had positive indirect effects on grain yield through the total biomass and the number of nodes that had pods. This was also supported by the highly significant positive correlation of most yield component characters, such as number of nodes that had pods, number of pod per plant, number of pods per node, total biomass, number of nodes per plant, thousand seed weight, and primary branches to grain yield. Likewise, the direct effect of harvest index and the indirect effect of number of pods per plant, number of branches per plant, number of seeds per pod, plant height and hundred seed weight for yield was also reported in path analysis study of faba bean (Kumar et al. 2013). However, chocolate spot disease and days to flowering had negative correlation with grain yield suggesting that late flowering cultivars were generally low yielding compared to early flowering cultivars. In the same way, Tadesse et al. (2011) reported negative direct effect of days to flowering on seed yield of faba bean. Despite the number of primary branches per plant having a high and positive correlation (0.72) with grain yield, it had negative and significant direct effect on grain. This contradiction seems to be due to the negative indirect effect of number of pods per node, number of pod per plant, number of seed per pod, number of nodes per plan, number of nodes that had pods through their effect on number of primary branches per plant. This led to significant negative direct effects of number of primary branches per plant to grain yield.

\section{Materials and methods}

\section{Faba bean germplasm}

Ten faba bean parental lines with different levels of resistance for chocolate spot $(\mathrm{CH})$ disease were obtained from Holetta Agricultural Research Center (HARC) and International Center for Agricultural Research in the Dry Area (ICARD) (Table 9). The parental lines were crossed in a $10 \times 10$ full-diallel mating scheme to form $90 \mathrm{~F}_{1}$ progenies including the reciprocals (Beyene et al., 2016).

\section{Experimental sites}

The experiments were conducted at three major faba beangrowing locations in the highlands of Ethiopia. The sites included Holetta Agricultural Research Centre (HARC) $\left(09^{\circ} 03^{\prime} \mathrm{N}, 38^{\circ} 30^{\prime} \mathrm{E}\right.$, and 2390 metres above sea level (m a.s.l). The average maximum and minimum temperatures at HARC during the growing period were $22.5^{\circ} \mathrm{C}$ and $8.5^{\circ} \mathrm{C}$, respectively, and the average rainfall received was $533.6 \mathrm{~mm}$, and soil $\mathrm{pH}$ of 4.62. The soil type at HARC experimental fields is Eutric Nitosols. The second site was the Kulumsa Agricultural Research Centre (KARC) $\left(08^{\circ} 01\right.$ 'N; 39 $09^{\circ} \mathrm{E}$; $2200 \mathrm{~m}$ a.s.l). The average maximum and minimum temperatures at KARC were $22.5^{\circ} \mathrm{C}$ and $10.9^{\circ} \mathrm{C}$, respectively. The average rainfall received was $584.1 \mathrm{~mm}$ and the soil $\mathrm{pH}$ was 5.2. The soil type of KARC experimental fields is xerosols. The third site was the Kofele $\left(07^{\circ} 04^{\prime} \mathrm{N}\right.$; $38^{\circ} 48^{\prime} \mathrm{E}$; $2660 \mathrm{~m}$ a.s.1.) and the average maximum and minimum temperatures were $18.3^{\circ} \mathrm{C}$ and $2.3^{\circ} \mathrm{C}$, respectively. The average rainfall received was $1077.4 \mathrm{~mm}$ with a soil $\mathrm{pH}$ of 4.37 .

\section{Experimental design and management}

The 100 entries consisting of $90 \mathrm{~F}_{1}$ reciprocal progenies (i.e., 45 progenies and their reciprocals) developed from the $10 \mathrm{x}$ 10 full diallel mating design and the 10 parental lines were planted in a $10 \times 10 \alpha$ - lattice design in single row plots of $3.0 \mathrm{~m}$ at $0.4 \mathrm{~m}$ inter-row and $0.06 \mathrm{~m}$ intra-row spacing. To minimize inter-plot interference, each test genotype was bordered by a common susceptible faba bean variety NC 58 , which also acted as a disease spreader. Fertilizer was applied $100 \mathrm{~kg} \mathrm{ha}^{-1}$ diammonium phosphate (DAP), i.e. $20 \mathrm{~kg} \mathrm{ha}^{-1}$ phosphorus and $18 \mathrm{~kg} \mathrm{ha}^{-1}$ nitrogen at planting. The trials were kept weed-free by hand weeding. In addition, trials were artificially inoculated with Botrytis fabae isolate with a spore concentration of $5 \times 10^{5} \mathrm{ml}^{-1}$ at 60 days from planting, which coincided with the flowering stage at all sites. Previous researchers (Bernard et al. 2006) recommended this practice. Inoculation was made during the late afternoon to avoid the effect of sunlight on spore viability.

\section{Data collection}

Data for the following agronomic traits were recorded on a plot basis at all location: days to $50 \%$ flowering (DTF) and days to physiological maturity (DTM) for the entire plot. Fifteen plants per genotype were randomly selected to record the data on number of primary branches (BB), height from ground to the first pod (HFP), number of pods per node (NPPN), number of pods per plant (NPPP), number of nodes that had pods (NWP), number of nodes per plant (NNP), number of seeds per pod (NSPP) and plant height (PH). Data total biomass (BM) at harvest, hundred seed weight (HSW) from randomly selected hundred-seeds from each plot and grain yield was taken from the entire plot. Grain yield $t$ ha $^{-1}$ data was taken from the entire plot. Grain yield was adjusted to $10 \%$ moisture content following the oven drying method. The total biomass and grain yield recorded on a plot basis was converted to $\mathrm{t} \mathrm{ha}^{-1}$ for statistical analysis.

Genotype reaction to Botrytis fabae was evaluated 7 days after inoculation and disease severity scoring was done six times on a weekly basis (7-day interval) from the same five randomly pre-tagged plants per genotype. In addition, general disease assessment of the genotypes for disease severity of chocolate spot was recorded from the whole plot once, at 88 days after planting. The severity of chocolate spot was recorded as a percentage of leaf area infected using the rating of Bernier et al. (1993) and Bernard et al. (2006). The scale is as follows: $1 \%$ - no disease symptoms or very small specks (highly resistant); 3\% - few small disease lesions (highly resistant); $6 \%$ - small coalesced lesions with some defoliation (resistant); $12 \%$ - large coalesced sporulating lesions, 20\% defoliation (moderately resistant); 25\% - large 
coalesced sporulating lesions, $50 \%$ defoliation and some dead plants (susceptible); and 50\% - extensive, heavy sporulation, stem girdling, blackening and death of more than $80 \%$ of the plants (highly susceptible).

\section{Data analysis}

The disease severity score data collected weekly in the field was used to calculate the area under the disease progress curve (AUDPC). Area under the disease progress curve (AUDPC) which is the most common mathematical tool used to model plant disease epidemics (Contreras-Medina et al., 2009) was calculated for each genotype using the disease severity score according to the following formula proposed by Shaner and Finney (1977).

$A U D P C=\sum_{i=1}^{n} 1 / 2[(y i+1+y i)(x i+1-x i)]$

Where: $y i=$ the cumulative disease severity percentage of infected plants at the $i^{\text {th }}$ observation (day $\mathrm{i}$ ), $\mathrm{xi}=$ time (days) at the $\mathrm{i}^{\text {th }}$ observation, $\mathrm{n}=$ total number of symptom observations.

AUDPC was calculated using Microsoft excel. The estimate of AUDPC was normalized by dividing with the total area of the graph (i.e. the number of days between the first and the last readings multiplied by maximum potential AUDPC), for a better visual comparison among genotypes over location (Mohapatra et al., 2008). The normalized AUDPC was referred to as the relative area under disease progress curve (rAUDPC).

\section{ANOVA of heterosis for chocolate spot resistance and yield:}

\section{Gardner - Eberhart II analysis}

Analyses of variance (ANOVA) to detect differences among the 100 entries for each trait in the study were separately performed on the data collected across environment. An inference was made from diallel cross mating technique about heterosis and its partitions; average heterosis, variety heterosis, and specific heterosis among the ten genotypes for chocolate spot resistance and yield in SAS (SAS Institute, 2012). The following models were used to determine the sums of squares for the analysis following the notation of Gardner and Eberhart:

$$
\left.\mathrm{X}_{\mathrm{jj}}{ }^{\prime}=\mathrm{u}+(1 / 2)\left(\mathrm{v}_{\mathbf{j}}+\mathrm{v}_{\mathbf{j}}\right)^{\prime}\right)=\left(\mathrm{B}^{\prime} \mathrm{G}\right)_{1}
$$

(iii) $\quad \mathrm{X}_{\mathrm{jj}},=\mathrm{u}+(1 / 2)\left(\mathrm{v}_{\mathbf{j}}+\mathrm{v}_{\mathbf{j}^{\prime}}\right)+v \mathrm{~h}+v\left(\mathrm{~h}_{\mathbf{j}}+\mathrm{h}_{\mathbf{j}^{\prime}}\right)=\left(\mathrm{B}^{\prime} \mathrm{G}\right)_{3}$

(iv) $\quad \mathrm{X}_{\mathbf{j} j},=\mathrm{u}+(1 / 2)\left(\mathrm{v}_{\mathbf{j}}+\mathrm{v}_{\mathbf{j}},\right)+v \mathrm{~h}+v\left(\mathrm{~h}_{\mathbf{j}}+\mathrm{h}_{\mathbf{j}}{ }^{\prime}\right)+v \mathrm{~s}_{\mathbf{j} j},=$

$\left(\mathrm{B}^{\prime} \mathrm{G}\right)_{4}$

In each of the models, $u, v_{j}, h$, and $s_{j j}$, indicate the mean and variety and heterosis effects. The coefficient $v$ in these models is zero when $j=j$ ' and one when $j \neq j$ '. Since the phenomenon of heterosis is important, the analysis maximizes the information on variety performance and the expression of heterosis of their crosses (Hallauer et al., 2010). Estimates of the variety and heterosis effects can be determined for each of the constants in the models. Data from the Gardner and Eberhart models II were analysed using the DIALLEL-SAS05 (Zhang et al., 2005) in SAS (SAS Institute, 2012). In general, partitions of entry sum of squares were as follows:

$\mathrm{Xij}=\mathrm{U}+1 / 2(V i+V j)+v h+v(\mathrm{hi}+\mathrm{hj})+v \mathrm{Sij} ; \mathrm{U}=$ mean effects; $\mathrm{Vj}=$ variety effects; $\mathrm{h}=$ average heterosis effects (Parents vs. Crosses); hi and hj $=$ variety heterosis effects; $\mathrm{Sij}$ $=$ specific heterosis effects for the cross $\mathrm{I} \times \mathrm{J} ; v=$ coefficient ranging from $0-1$ when $v=0$ then $\mathrm{i}=\mathrm{j}$ (it's a self), when $v=1$ then $\mathrm{i} \neq \mathrm{j}$.

Heterosis was also used to examine heterotic relationships among the lines (Falconer and Mackay, 1996). Mid-parent heterosis (MPH) determined using the mid-parent (MP) calculated as: $\mathrm{MPH}=\frac{\mathrm{F}_{1}-\mathrm{MP}}{\mathrm{MP}} \times 100$ where $\mathrm{F}_{1}$ is the performance of hybrids, $\mathrm{MP}=\frac{\left(\mathrm{P}_{1}+\mathrm{P}_{2}\right)}{2}$ in which $\mathrm{P}_{1}$ and $\mathrm{P}_{2}$ are the performance values of parents, respectively. Better-parent heterosis (BPH) (Heterobeltiosis) (\%) was calculated as: $\mathrm{BPH}=\frac{\mathrm{F}_{1}-\mathrm{BP}}{\mathrm{BP}} \times 100$ where $\mathrm{F}_{1}=$ mean of the $\mathrm{F}_{1}$ progenies performance, $B P=$ the mean of the better-parent /superior parent (Sleper and Poehlman, 2006). Path co-efficient analysis among the yield and yield component traits was done using PATHSAS: the SAS computer program for path coefficient analysis of quantitative data as described by Cramer and Wehner (1999) in SAS (SAS Institute, 2012).

\section{Conclusion}

In conclusion, the high mid and better-parent heterosis for grain yield observed in the crosses NC58 x ILB-4726, ILB$4726 \times$ Kasa, NC58 $\times$ BPL-710, ILB-938 $\times$ CS-20-DK, ILB$938 \times$ CS-20-DK and CS-20-DK $\times$ BPL-710 suggests that these hybrids could be further considered in the faba bean breeding programme aiming both for segregant breeding and hybrid development in the long term. The best hybrids; ILB$4726 \times$ Kasa, ILB-4726 $\times$ Bulga-70, CS-20-DK $\times$ Gebelcho, NC58 $\times$ ILB-4726, Kasa $\times$ BPL-710 and ILB-938 $\times$ Kasa, that exhibited negative heterosis over the mid and betterparent for chocolate spot resistance could be considered as source of segregants in faba bean breeding for disease resistance. The three crosses ILB-4726 $\times$ Kasa, ILB-4726 $\times$ Bulga-70, NC58 $\times$ ILB-4726 are recommended for faba bean breeding programme aiming for grain yield and chocolate spot disease resistance. Correlation and path coefficients analysis indicated that the number of nodes that had pods and total biomass were major contributors through their direct effects on grain yield. Therefore, these two traits should be given high weight in selection indices for faba bean breeding.

\section{Acknowledgements}

The authors are grateful to the African Centre for Crop Improvement (ACCI) and the Alliance for a Green Revolution in African (AGRA) for financial support to first author. The Ethiopian Institute of Agricultural Research (EIAR) and Holetta Agricultural Research Centre (HARC) are gratefully acknowledged for hosting the study and providing research facilities.

\section{References}

Alghamdi SS (2009) Heterosis and combining ability in a diallel cross of eight faba bean (Vicia faba L.) genotypes. Asian J Crop Sci. 1:66-76.

Amini Z, Khodambashi M, Houshmand S (2013) Correlation and path coefficient analysis of seed yield related traits in maize. Int J Agric Crop Sci. 5:2217-2220.

Azarpour E, Bidarigh S, Moraditochaee M, Danesh RK, Bozorgi HR, Bakian M (2012) Path Coefficient Analysis of Seed Yield and its Components in Faba Bean (Vicia faba L.) under Nitrogen and Zinc Fertilizer Management. Int J Agric Crop Sci. 4: $1559-1561$.

Bernard T, Baranger A, Carmen MA, Sabine B, Martin B, Weidong C, Jenny D, Kurt L, Mohanned K, Diego R, Mohamed S, Josefina SC, Mark S, Fred JM (2006) Screening techniques and sources of resistance to foliar diseases caused 
by major necrotropic fungi in grain legumes. Euphytica. 147 (1) :223-253.

Bernier CC, Hanounik SB, Hussein MM, Mohamed HA (1993) Field manual of common Faba bean diseases in the Nile Vally. International Centre for Agricultural Research in the Dry Areas (ICARDA). Information Bulletin No. 3.

Beyene A, John D, SibiyaJ, Fikre A (2016) Gene action determining grain yield and chocolate spot (Botrytis fabae) resistance in a faba bean. Euphytica. 207: 293-304.

Bond DA (1987) Recent developments in breeding field beans (Vicia faba L.). Plant Breeding 99:1-26.

Bond DA, Lawes DA, Hawtin GC, Saxena MC, Stephens JS (1985) Faba Bean (Vicia faba L.). In: R. J. Summerfield and E. H. Roberts, editors, Grain legume crops. William Collins \& Sons Co. Ltd., London, UK.

Contreras-Medina LM, Torres-Pacheco I, Guevara-González RG, Romero-Troncoso RJ, Terol-Villalobos IR, Osornio-Rios RA (2009) Mathematical modeling tendencies in plant pathology. Afr J Biotec. 8:7399-7408.

Cramer CS, Wehner TC (1999) PATHSAS: A SAS Computer Program for Path Coefficient Analysis of Quantitative Data. J Herd. 90:260.

Das A, Pandey S, and Dasgupta T (2013) Association of heterosis with combining ability and genetic givergence in sesame (Sesamum Indicum L.). Int J Sci Technol Res. 2:307314.

Dewey D, Lu KH (1959) A correlation and path-coefficient analysis of components of crested Wheat grass seed production. Agron J. 51:515-518.

Dhuppe MV, Madrap IA, Chandankar GD, More SS (2005) Correlation and path analysis in mung bean (Vigna radiata $\mathrm{L}$. Wilezeck). J Soils Crops. 15:84-89.

Duvick DN (1999) Heterosis: feeding people and protecting natural resources. In: J. G. Corrs, editor The genetics and exploitation of heterosis in crops. Pandey S. Madison Wisconsin: American Society of Agronomy, Incorporated. p19-29.

El-Hady MM, Olaa SMA, El-Galaly AM, Salem MM (2006) Heterosis and combining ability analysis of some faba bean genotypes. J Agric Res Tanta U. 32:134-148.

Falconer DS, Mackay TFC (1996) Introduction to Quantitative Genetics. $4^{\text {th }}$ ed. Longman group Ltd, London UK.

Farag HIA, Afiah SA (2012) Analysis of gene action in diallel crosses among some faba bean (Vicia faba L.) genotypes under Maryout conditions. Ann Agr Sci. 57(1): 37-46

Farshadfar M, Farshadfar E (2008) Genetic variability and path analysis of chickpea (Cicer arientinum L.) Landraces and lines. J Appl Sci. 8(21): 3951-3956.

FAOSTAT ( 2016) Data base. Available at: http://faostat.fao.org/beta/en/\#data/QC

Fehr WR (1987) Principles of Cultivar Development, Vol. 2: Crop Species. Macmillan USA, New York.

Ferris S, Kaganzi E (2008) Evaluating marketing opportunities for haricot beans in Ethiopia. IPMS (Improving Productivity and Market Success) of Ethiopian Farmers Project Working Paper 7. ILRI (International Livestock Research Institute), Nairobi, Kenya. p68.

Fu D, Xiao M, Hayward A, Fu Y, Liu G, Jiang G, Zhang H. (2014) Utilization of crop heterosis: a review. Euphytica 197:161-173.

Garud CB, Borgaonkar SB, Chinchane BN (2014) Correlation and path analysis of seed quality characters in Soybean. Indian J Plant Sci. 9:293-294.

Gnanasambandam A, Paull J, Torres A, Kaur S, Leonforte T, Li $\mathrm{H}$, Zong X, Yang T, Materne M(2012) Impact of molecular technologies on faba bean (Vicia faba L.) breeding strategies. Agronomy 2:132-166.
Haciseferogullari H, Gezer I, Bahtiyarca Y, Menges HO (2003) Determination of some chemical and physical properties of Sakız faba bean (Vicia faba L. Var. major). J Food Eng. 66:475-479

Hallauer AR, Carena MJC, Miranda JB, Filho (2010) Hand book of plant Breeding: Quantitative Genetics in Maize Breeding. Springer New York, Dordrecht Heidelberg London.

Hanounik SB, Robertson LD (1989) Resistance in Vicia faba germplasm to blight caused by Ascochyta fabae. Plant Dis. 73:202-205

Jat BL, Jakhar ML (2014) Phenotypic path co-efficient analysis in Taramira (Eruca sativa L.). Int J Plant Sci. 9:231-233.

Jatoi WA, Baloch MJ, Khan NU, Munir M, Khakwani AA, Vessar NF, Panhwar SA, Gul S (2014) Heterosis for yield and physiological traits in wheat under water stress conditions. J Anim Plant Sci. 24:252-261.

Kumar VI, Verma PN, Yadav CB (2013) Correlation and Path Coefficient Analysis in Faba Bean (Vicia faba L.) under Irrigated Condition. Trends Biosci. 6 (5):576-578.

Lim SM, White DG (1978) Estimates of heterosis and combining ability for resistance of maize to Colletotrichum graminicola. Phytopathology 68:1336-1342.

Lindemann WC, Glover CR (2003) Nitrogen fixation by legumes In: Cooperative Extension Service Guide A-129, editor New Mexico State University and the USDA.

Link W, Schill B, Kittlitz EV (1996) Breeding for wide adaptation in faba bean. Euphytica 92:185-190.

Matiello RR, Brunelli KR, Gomes Lopes MT, Coêlho Morello RMS, Silva HS, Camargo LEA (2012) Inheritance of resistance to anthracnose stalk rot (Colletotrichum graminicola) in tropical maize inbred lines. Crop Breed Appl Biot. 12:179184

Mohapatra NK, Mukherjee AK, Suriya Rao AV, Nayak P (2008) Disease progress curves in the rice blast pathosystem compared with the logistic and gompertz models. ARPN J Agric Biol Sci. 3:28-37.

Munaro EM, Eyherabide GH, D'Andrea KE, Cirilo AG, Otegui ME (2011) Heterosis $\times$ environment interaction in maize: What drives heterosis for grain yield? Field Crops Res 124:441-449.

SAS Institute ( 2012) SAS proprietary software. Release 9.3 SAS Inst., Cary, NC, USA

Shaner G, Finney FE (1977) The effect of nitrogen fertilization on the expression of slow-mildewing in Knox wheat. Phytopathology 67:1051-1056.

Sincik M, Goksoy AT (2014) Investigation of correlation between traits and path analysis of confectionary sunflower genotypes. Not Bot Horti Agrobot Cluj Napoca. 42(1): $227-$ 231

Sleper DA, Poehlman JM (2006) Breeding field crops 5th ed. Iowa State Avenue. Blackwell Ames..

Suso MJ, Moreno MT, Melchinger AE, (1999) Variation in outcrossing rate and genetic structure on six cultivars of Vicia faba L. as affected by geographic location and year. Plant Breeding 118 (4): 347-350

Tadesse T, Fikere M, Legesse T, Parven A (2011) Correlation and path coefficient analysis of yield and its component in faba bean (Vicia faba L.) germplasm. Int J Biodivers Conserv. 3(8):376-382.

Tiruneh MA, Ali HM, Zelleke H (2013) Estimation of better parent and economic heterosis for yield and associated traits in common beans. J Appl Biosci. 711:5706-5714.

Zeid M, Schon CC, Link W (2004) Hybrid performance and AFLP- based genetic similarity in faba bean. Euphytica 139:207-216

Zhang Y, Kang MS, Lamkey KR (2005) Diallel-SAS05: A comprehensive program for Griffing's and Gardner-Eberhart analyses. Agron J. 97:1097-1106. 